Pure and Applied Mathematics Quarterly

Volume 3, Number 3

(Special Issue: In honor of

Leon Simon, Part 2 of 2$)$

$631-645,2007$

\title{
Relative Mapping Class Group of the Trivial and the Tangent Disk Bundles over the Sphere
}

\author{
Nikolai A. Krylov
}

\begin{abstract}
In this paper we determine the group of pseudo-isotopy classes of diffeomorphisms of the trivial and the tangent unit disk bundles to the standard sphere, which restrict to the identity map on the boundary.
\end{abstract}

\section{INTRODUCTION}

Let $M$ be an oriented connected smooth manifold with non-empty boundary $\partial M$. We consider the group of pseudo-isotopy classes of diffeomorphisms of $M$ which restrict to the identity map on $\partial M$. Obviously, such diffeomorphisms preserve orientation. Recall that two diffeomorphisms $f_{0}, f_{1} \in \operatorname{Diff}(M)$ which keep $\partial M$ pointwise fixed are called pseudo-isotopic (rel boundary) if there exists a diffeomorphism $\Phi: M \times I \longrightarrow M \times I$ such that $\left.\Phi\right|_{M \times\{0\}}=f_{0},\left.\Phi\right|_{M \times\{1\}}=f_{1}$ and $\left.\Phi\right|_{\partial M \times I}=I d$. We write $f_{0} \sim f_{1}$ to indicate that $f_{0}$ is pseudo-isotopic to $f_{1}$. If $\operatorname{dim}(M)=2$, the group is known as the classical mapping class group of a surface. We will also call the group of pseudo-isotopy classes of diffeomorphisms of $M$ which are fixed on the boundary as the (relative) mapping class group and denote it by $\tilde{\pi}_{0} \operatorname{Diff}(M$, rel $\partial)$. For the handlebodies in general, the pseudoisotopy classes of diffeomorphisms with no constraint on the boundary had been studied by Wall in [26]. There are also explicit computations by Sato (see [22], Theorem 1.7) for some cases of the trivial disk bundle over the standard sphere. The corresponding group of pseudo-isotopy classes of diffeomorphisms with no

Received July 28, 2005 .

2000 Mathematics Subject Classification. Primary 57R50, 57R52. 
constraint on the boundary is denoted by $\tilde{\pi}_{0} \operatorname{Diff}(M)$. In principle, one could approach the study of the homotopy type of the space of smooth (as well as piecewise linear and topological) automorphisms of a manifold $M$ via surgery theory and the block $h$-structure space $\tilde{\mathcal{S}}_{d}^{s}(M)$. The block structure space was considered, for instance, by Quinn in [19] and has the property (in the case of smooth automorphisms):

$$
\pi_{k}\left(\tilde{\mathcal{S}}_{d}^{s}(M)\right) \cong \mathbb{S}^{d}\left(M \times \Delta^{k}, \partial\right)
$$

where $\mathbb{S}^{d}\left(M \times \Delta^{k}, \partial\right)$ is the set of smooth structures on $M \times \Delta^{k}$ which induce diffeomorphisms on the boundary $\partial\left(M \times \Delta^{k}\right)$ (cf. $\S 10$ of [28]). The following homotopy fiber sequence links this block $h$-structure space to the block automorphism space $\widetilde{\operatorname{Diff}}(M)$ :

$$
\widetilde{\operatorname{Diff}}(M) \longrightarrow G^{s}(M) \longrightarrow \tilde{\mathcal{S}}_{d}^{s}(M) .
$$

Using, for example, the homotopy exact sequence of this fibration one can compute $\tilde{\pi}_{0} \operatorname{Diff}\left(M \times D^{k}\right.$, rel $\left.\partial\right) \cong \pi_{k} \widetilde{\operatorname{Diff}}(M)$. The reader will find details and references on this account in a survey paper [29] by Weiss and Williams.

The present work is devoted to computations of the relative mapping class group of the trivial and the tangent disk bundles over the standard sphere using "more explicit" geometric methods. I will discuss first the trivial disk bundle over $S^{p}$, and will use the results of Levine [13] and Sato [21] who determined the mapping class group of $S^{p} \times S^{q}$ up to extension (cf. also [24]). In $\S 2.2$ below, I will indicate how one could use surgery theory and the ideas mentioned above (thanks go to the referee) to compute $\tilde{\pi}_{0} \operatorname{Diff}\left(S^{1} \times D^{q}\right.$, rel $\partial$ ), when $q \geq 4$. Then I will discuss the relative mapping class group of the closed tangent disk bundle over the sphere and will use well known results on the monodromy of the quadratic singularity of the complex hypersurface defined by $f(z)=z_{0}^{2}+z_{1}^{2}+\ldots+z_{p}^{2}$ (see [14] for the details). Integer coefficients are understood for all homology groups, unless otherwise stated, and symbols $\simeq$ and $\cong$ are used to denote respectively a homotopy equivalence and an isomorphism (in geometric and algebraic categories). I will follow notations of [21] and denote by $\tilde{\pi}_{0} \operatorname{SDiff}\left(S^{p} \times S^{q}\right)$ the subgroup of $\tilde{\pi}_{0} \operatorname{Diff}\left(S^{p} \times S^{q}\right)$ which consists of classes with representatives that induce trivial action on the homology. The $h$-cobordism classes of all homotopy $m$-spheres form an abelian group under the connected sum operation and we denote such a group by $\Theta_{m}$ (see [10] for details). 
Acknowledgement: I am very thankful to the referee who has made a few useful suggestions on the exposition, and explained to me how one could use surgery theory to compute the relative mapping class group of $S^{p} \times D^{q}$.

$$
\text { 2. } \tilde{\pi}_{0} \operatorname{Diff}\left(S^{p} \times D^{q}, \text { rel } \partial\right)
$$

2.1. Relative mapping class group and the double. Given a manifold $M$ with non-empty boundary, one can consider the double $\mathcal{D} M$ of $M$ defined as $\mathcal{D} M:=\partial(M \times I) . \mathcal{D} M$ is a closed manifold with the canonically defined smooth structure (cf. [18]). Since $\partial(M \times I) \cong M \times\{0\} \cup(\partial M) \times I \cup M \times\{1\}$ and $(\partial M) \times I \cup M \times\{1\} \cong M$ (which we will denote by $M_{+}$), one can also think of the double as of the union of two copies of $M$ glued together along the boundary via the identity map:

$$
\mathcal{D} M \cong M \cup M_{+}
$$

For example, if $M \cong S^{p} \times D^{q}$ then $\mathcal{D} M \cong S^{p} \times S^{q}$. Take $\varphi \in \operatorname{Diff}(M$, rel $\partial)$, then one can use the identity to extend $\varphi$ to a diffeomorphism $\tilde{\varphi}$ of $\mathcal{D} M$. To be more precise, we define

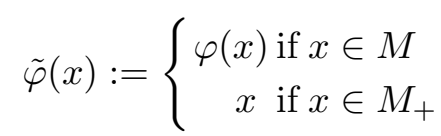

This construction gives a map $\operatorname{Diff}(M$, rel $\partial) \longrightarrow \operatorname{Diff}(\mathcal{D} M)$, which induces a homomorphism $\omega: \tilde{\pi}_{0} \operatorname{Diff}(M, \operatorname{rel} \partial) \longrightarrow \tilde{\pi}_{0} \operatorname{Diff}(\mathcal{D} M)$ defined by $\omega([\varphi]):=[\tilde{\varphi}]$. The following proposition generalizes Theorem 2 of [9].

\section{Proposition 1.}

Homomorphism $\omega: \tilde{\pi}_{0} \operatorname{Diff}(M$, rel $\partial) \rightarrow \tilde{\pi}_{0} \operatorname{Diff}(\mathcal{D} M)$ is injective for all $M$.

Proof. If $\varphi \in \tilde{\pi}_{0} \operatorname{Diff}(M$, rel $\partial)$ and $\tilde{\varphi} \sim I d$, then there exists an extension $\Phi \in$ $\operatorname{Diff}(M \times I)$ of $\tilde{\varphi} \in \operatorname{Diff}(\partial(M \times I))$. Since $\Phi$ is equal to $\varphi$ on $M \times\{0\}$ and the identity map on $\partial(M) \times I \cup M \times\{1\}$, this $\Phi$ is a relative pseudo-isotopy that connects $\varphi$ with $I d$.

Remark: There exist infinitely many contractible 4-manifolds $W$ with $\partial W$ irreducible (e.g. some homology 3 -sphere with a nontrivial fundamental group), and such that $\mathcal{D} W \cong S^{4}$ (see, for example, $\S 6$ of [6]). Since $\Theta_{5} \cong\{1\}$, we immediately obtain 
Corollary 1. Let $W^{4}$ be a contractible manifold, such that $\mathcal{D} W$ is diffeomorphic to $S^{4}$, with $\partial W$ irreducible. Then $\tilde{\pi}_{0} \operatorname{Diff}(W$, rel $\partial) \cong\{1\}$.

Let us assume now that $M=S^{p} \times D^{q}$. It is easy to see that when $p \neq q$, the diffeomorphism $(x, y) \longmapsto\left(R_{p}(x), R_{q}(y)\right)$ (where $(x, y) \in S^{p} \times S^{q}$ and $R_{p}$ and $R_{q}$ denote the reflections of $S^{p}$ and $S^{q}$ respectively) generates the quotient group $\tilde{\pi}_{0} \operatorname{Diff}\left(S^{p} \times S^{q}\right) / \tilde{\pi}_{0} \operatorname{SDiff}\left(S^{p} \times S^{q}\right) \cong \mathbb{Z}_{2}$ (cf. [13], §1.2). Since the extension diffeomorphism $\tilde{\varphi}$ fixes $S^{p} \times D_{+}^{q}=M_{+} \subset \mathcal{D} M$, it follows from Proposition 2.1 of [21] and proposition above that $\tilde{\pi}_{0} \operatorname{Diff}\left(S^{p} \times D^{q}\right.$, rel $\left.\partial\right)$ must be a subgroup of $\tilde{\pi}_{0} \operatorname{SDiff}\left(S^{p} \times S^{q}\right)$.

Suppose that $1 \leq p<q$ and $3 \leq q$, or $4 \leq p=q$, then there is a homomorphism

$$
B: \tilde{\pi}_{0} \operatorname{SDiff}\left(S^{p} \times S^{q}\right) \longrightarrow \pi_{p}(S O(q+1)) .
$$

defined by Sato as follows (cf. [21], §3): Take a representative $f$ of a class $[f] \in \tilde{\pi}_{0} \operatorname{SDiff}\left(S^{p} \times S^{q}\right)$ and pick a point $z \in S^{q}$. Then $S^{p}=S^{p} \times z \subset \mathcal{D} M$ will represent a generator of $H_{p}(\mathcal{D} M) \cong \mathbb{Z}$. Since $f$ acts trivially on $H_{p}(\mathcal{D} M)$, it follows from the Hurewicz theorem and the result of Haefliger [7] that there exists a smooth isotopy from $f$ to $f^{\prime}$, where $f^{\prime}$ is the identity on $S^{p} \times z$. Furthermore, if we take the tubular neighborhood $S^{p} \times D^{q}$ of the sphere $S^{p} \times z$, then by the tubular neighborhood theorem we can assume that $f^{\prime}$ is isotopic to $f^{\prime \prime}$ such that $f^{\prime \prime}(x, y)=(x, b(f) \cdot y)$, where $(x, y) \in S^{p} \times D^{q}$ and $b(f)$ is a smooth map $S^{p} \longrightarrow S O(q)$. Denote the inclusion map $S O(q) \hookrightarrow S O(q+1)$ by $S$ and the homotopy class of $b(f)$ by $[b(f)] \in \pi_{p}(S O(q))$, then $B$ is defined by the formula:

$$
B([f]):=S_{*}([b(f)]) .
$$

For each element $[f] \in \tilde{\pi}_{0} \operatorname{Diff}\left(D^{m}, \operatorname{rel} \partial\right) \cong \tilde{\pi}_{0} \operatorname{Diff}\left(S^{m}\right) \cong \Theta_{m+1}$ one defines a diffeomorphism $\iota_{r}(f) \in \operatorname{Diff}\left(S^{p} \times D^{m-p}\right.$, rel $\left.\partial\right)$ as the identity map outside an embedded disk $\mathbb{D}^{m} \hookrightarrow \operatorname{Int}\left(S^{p} \times D^{m-p}\right)$ and as $\left.f\right|_{D^{m}}$ on this $\mathbb{D}^{m}$ (see $\S 4$ of [21] for the details). It is easily deduced from $\S 4$ of [21] that this construction induces a monomorphism $\iota_{r}: \Theta_{p+q+1} \hookrightarrow \tilde{\pi}_{0} \operatorname{Diff}\left(S^{p} \times D^{q}\right.$, rel $\left.\partial\right)$.

Furthermore, let us denote by $\mathrm{FC}_{q}^{p+1}$, the group of pseudo-isotopy classes of orientation preserving embeddings of $S^{q} \times D^{p+1}$ in $S^{q+p+1}$. Such groups were introduced by Haefliger and Levine and the reader is referred to $\S 5$ of [8] or to [12] for the details. For example, $\mathrm{FC}_{q}^{p+1} \cong \pi_{q}(S O(p+1))$, when $q<2 p$ (see 
[8], Corollaries $5.9 \& 6.6)$. These groups could also be understood in terms of homotopy groups of the spaces $G\left(S^{p}\right), G$ and $O$.

\section{Lemma 1.}

$$
\tilde{\pi}_{0} \operatorname{Diff}\left(S^{p} \times D^{q}, \text { rel } \partial\right) \cong \begin{cases}\Theta_{p+q+1} \oplus \mathrm{FC}_{q}^{p+1} & \text { if } 1<p<q \\ \Theta_{p+q+1} \oplus \pi_{q}(S O(p+1)) & \text { if } 1<q<p\end{cases}
$$

Proof. Assume first that $p<q$, then we have the exact sequence (see [21], Theorem II or [13], Theorem 2.4):

$$
0 \longrightarrow \mathrm{FC}_{q}^{p+1} \oplus \Theta_{p+q+1} \longrightarrow \tilde{\pi}_{0} \operatorname{SDiff}\left(S^{p} \times S^{q}\right) \stackrel{B}{\longrightarrow} \pi_{p}(S O(q+1)) \longrightarrow 0 .
$$

Since the diffeomorphism $\tilde{\varphi}$ fixes $S^{p} \times D_{+}^{q}$, it is clear from the definition of $B$ that $B([\tilde{\varphi}])=\{0\}$, i.e. $\tilde{\pi}_{0} \operatorname{Diff}\left(S^{p} \times D^{q}\right.$, rel $\left.\partial\right) \subset \operatorname{Ker}(B)$. Sato had shown ([21], Lemma 3.3) that for any element $[u] \in \operatorname{Ker}(B)$ one can find a representative $u \in \operatorname{Diff}\left(S^{p} \times S^{q}\right)$ such that $\left.u\right|_{S^{p} \times D_{+}^{q}}=I d$, and therefore $\tilde{\pi}_{0} \operatorname{Diff}\left(S^{p} \times D^{q}\right.$, rel $\left.\partial\right) \cong$ $\operatorname{Ker}(B)$.

Let now $p$ be larger than $q$. In this case $\operatorname{Im}(B)=\pi_{q}(S O(p+1))$ and for every class $[z] \in \pi_{q}(S O(p+1))$ we can choose a smooth representative

$$
r:\left(D^{q}, S^{q-1}\right) \longrightarrow(S O(p+1), I d)
$$

and define a relative diffeomorphism $\vartheta$ of $S^{p} \times D^{q}$ by the formula $\vartheta(x, y):=$ $(r(y) \cdot x, y)$, where $(x, y) \in S^{p} \times D^{q}$. Let us also use $\omega$ to denote the inclusion $\tilde{\pi}_{0} \operatorname{Diff}(M$, rel $\partial) \hookrightarrow \tilde{\pi}_{0} S \operatorname{Diff}(\mathcal{D} M)$, when every $\varphi \in \operatorname{Diff}(M$, rel $\partial)$ induces the trivial automorphism in homology of the double. Proof of Proposition 3.2 of [21] shows that the composition $B \circ \omega$ is a surjection. If we take an element $\left[\varphi_{0}\right] \in \tilde{\pi}_{0} \operatorname{Diff}(M$, rel $\partial)$ such that $B\left(\left[\tilde{\varphi}_{0}\right]\right)=\{1\}$, then propositions 5.2 and 5.3 of [21] imply that $\tilde{\varphi}_{0}$ (modulo some element of $\Theta_{p+q+1}$ if needed) can be extended to a diffeomorphism of $S^{p} \times D^{q+1}$, i.e. $\left[\tilde{\varphi}_{0}\right] \in \Theta_{p+q+1}$ and therefore $\tilde{\pi}_{0} \operatorname{Diff}\left(S^{p} \times\right.$ $D^{q}$, rel $\left.\partial\right) \cong \pi_{q}(S O(p+1)) \oplus \Theta_{p+q+1}$.

\section{Lemma 2.}

$$
\tilde{\pi}_{0} \operatorname{Diff}\left(S^{p} \times D^{q}, \text { rel } \partial\right) \cong \begin{cases}\{1\} & \text { if } p=1, q=2 \\ \Theta_{q+1} \oplus \Theta_{q+2} & \text { if } p=1, q \geq 3 \\ \Theta_{p+2} \oplus \mathbb{Z}_{2} & \text { if } q=1, p \geq 2\end{cases}
$$

Proof. $\tilde{\pi}_{0} \operatorname{Diff}\left(S^{1} \times D^{2}\right.$, rel $\left.\partial\right) \hookrightarrow \tilde{\pi}_{0} \operatorname{Diff}\left(S^{1} \times S^{2}\right)$ by our Proposition 1. Since $\tilde{\varphi} \in \operatorname{Diff}\left(S^{1} \times S^{2}\right)$ acts trivially on the homology, it follows from Theorem 5.1 of [5] 
that $\tilde{\varphi}$ is either pseudo-isotopic to the identity or the diffeomorphism $T$ of $S^{1} \times S^{2}$ defined by $T(t, x):=(t, f(t) \circ x)$ where $f: S^{1} \longrightarrow S O(3)$ is a smooth generator of $\pi_{1}(S O(3)) \cong \mathbb{Z}_{2}$. If we had $\tilde{\varphi} \sim T$, then $\tilde{\varphi}$ would extend to a diffeomorphism of $S^{1} \times D^{3}$, and therefore $\varphi$ would be pseudoisotopic to the identity map. Thus $\tilde{\pi}_{0} \operatorname{Diff}\left(S^{1} \times D^{2}\right.$, rel $\left.\partial\right) \cong\{1\}$.

Consider now $\tilde{\pi}_{0} \operatorname{Diff}\left(S^{1} \times D^{q}\right.$, rel $\left.\partial\right)$ with $q \geq 3$. Here we also can assume that $\tilde{\pi}_{0} \operatorname{Diff}\left(S^{1} \times D^{q}\right.$, rel $\left.\partial\right) \subset \operatorname{Ker}(B)$ and according to Proposition 6.3 of [21], the latter group is isomorphic to $\Theta_{q+1} \oplus \Theta_{q+2}$. For an element $[g] \in \tilde{\pi}_{0} \operatorname{Diff}\left(D^{q}\right.$, rel $\left.\partial\right) \cong$ $\tilde{\pi}_{0} \operatorname{Diff}\left(S^{q}\right) \cong \Theta_{q+1}$, we associate the diffeomorphism $G \in \operatorname{Diff}\left(S^{1} \times D^{q}\right)$ defined by the formula: $G(x, y):=(x, g(y))$. Thus we obtain a map $K: \Theta_{q+1} \rightarrow \tilde{\pi}_{0} \operatorname{Diff}\left(S^{1} \times\right.$ $D^{q}$, rel $\partial$ ) which is a monomorphism (see Proposition 6.2 of [21]). Since $\Theta_{q+2}$ is also a subgroup of $\tilde{\pi}_{0} \operatorname{Diff}(M$, rel $\partial)$, we see that $\tilde{\pi}_{0} \operatorname{Diff}\left(S^{1} \times D^{q}\right.$, rel $\left.\partial\right) \cong \Theta_{q+1} \oplus$ $\Theta_{q+2}$.

Gluck had shown in $[5]($ see $\S 8-\S 13)$ that $\tilde{\pi}_{0} \operatorname{Diff}\left(S^{2} \times D^{1}\right.$, rel $\left.\partial\right) \cong \mathbb{Z}_{2}$. As the generator of this group, one can take the homeomorphism $T$ of $S^{2} \times D^{1}$ defined by $T(x, t):=(f(t) \circ x, t)$, where $[f] \in \pi_{1}(S O(3))$ is as above. Since $\Theta_{4} \cong\{1\}$, we can assume for the rest of our proof that $p \geq 3$. Since $\tilde{\pi}_{0} \operatorname{Diff}(M$, rel $\partial) \hookrightarrow$ $\tilde{\pi}_{0} S \operatorname{Diff}(\mathcal{D} M)$ and using once again the generalized Dehn twist $(x, y) \rightarrow(\alpha(y) \circ$ $x, y) \in S^{p} \times I$ with $[\alpha]=$ a smooth generator of $\pi_{1}(S O(p+1))$, it is easy to see that $B \circ \omega$ is an epimorphism, i.e. $\operatorname{Im}(B \circ \omega)=\mathbb{Z}_{2}$. Take $\varphi \in \operatorname{Diff}\left(S^{p} \times D^{1}\right.$, rel $\left.\partial\right)$ such that $[\tilde{\varphi}] \in \operatorname{Ker}(B)$. According to Sato $([21], \S 6)$, we have the exact sequence

$$
0 \longrightarrow \Theta_{p+1} \stackrel{K}{\longrightarrow} \operatorname{Ker}(B) \stackrel{C}{\longrightarrow} \Theta_{p+2} \longrightarrow 0
$$

where map $K$ was just defined in the paragraph above and $C$ is the inverse map to the monomorphism $\iota_{r}$ which has been mentioned at the beginning (all the details regarding the homomorphism $C$ can be found in $\S 4$ of [21]). If we have $[\tilde{\varphi}] \in \operatorname{Ker}(C)$, then we can assume that there exists $f \in \operatorname{Diff}\left(S^{p} \times S^{1}\right)$ such that $f \sim \tilde{\varphi}$ and $f(x, y)=(g(x), y)$ with $[g] \in \tilde{\pi}_{0} \operatorname{Diff}\left(D^{q}\right.$, rel $\left.\partial\right) \cong \tilde{\pi}_{0} \operatorname{Diff}\left(S^{q}\right) \cong \Theta_{q+1}$. In this case we could extend $\tilde{\varphi}$ to a diffeomorphism of $S^{p} \times D^{2}$, i.e. $\tilde{\varphi} \sim I d$. Hence $\tilde{\pi}_{0} \operatorname{Diff}\left(S^{p} \times D^{1}\right.$, rel $\left.\partial\right) \cong \Theta_{p+2} \oplus \mathbb{Z}_{2}$.

2.2. Using Surgery Theory. Here we briefly indicate how one could approach the same problem via surgery theory. Consider the commutative diagram of the Sullivan-Wall long exact surgery sequences for the classification of smooth and 
PL structures on the product $S^{1} \times D^{q}=: M$, with $q \geq 4$ (see [15] or [28] for the details):

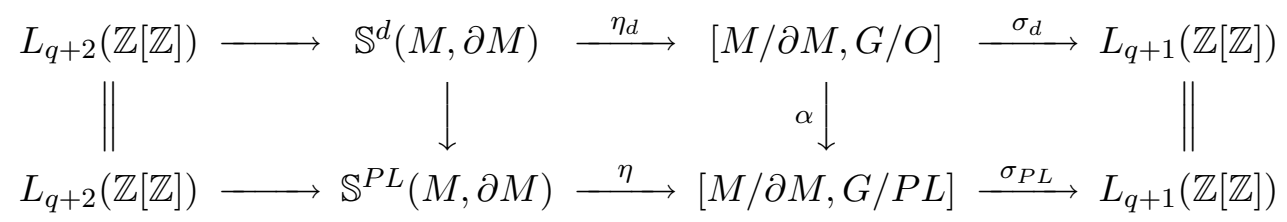

Since $\mathbb{S}^{P L}\left(S^{1} \times D^{q}, S^{1} \times S^{q-1}\right) \cong\{1\}$ (see Theorem 15A.2 of [28]), we see that $\operatorname{Im}\left(\eta_{d}\right) \cong \operatorname{Ker}(\alpha)$. Furthermore, using the basic property of the fibration

$$
P L / O \longrightarrow G / O \longrightarrow G / P L,
$$

and the obvious homotopy equivalence $S^{1} \times D^{q} / S^{1} \times S^{q-1} \simeq S^{q+1} \vee S^{q}$ we conclude that

$$
\mathbb{S}^{d}\left(S^{1} \times D^{q}, S^{1} \times S^{q-1}\right) \cong\left[S^{q+1} \vee S^{q}, P L / O\right] \cong \pi_{q+1}(P L / O) \oplus \pi_{q}(P L / O),
$$

and hence $\pi_{q+1}\left(\tilde{\mathcal{S}}_{d}^{s}\left(S^{1}\right)\right) \cong \pi_{q+2}(P L / O) \oplus \pi_{q+1}(P L / O)$. The homotopy fiber sequence

$$
\widetilde{\operatorname{Diff}}\left(S^{1}\right) \longrightarrow G^{s}\left(S^{1}\right) \longrightarrow \tilde{\mathcal{S}}_{d}^{s}\left(S^{1}\right)
$$

splits in the term $G^{s}\left(S^{1}\right) \simeq O(2)$, and we get the splitting in the corresponding homotopy long exact sequence. That implies

$$
\pi_{q}\left(\widetilde{\operatorname{Diff}}\left(S^{1}\right)\right) \cong \pi_{q+1}\left(\tilde{\mathcal{S}}_{d}^{s}\left(S^{1}\right)\right) \oplus \pi_{q}(O(2)) \cong \pi_{q+2}(P L / O) \oplus \pi_{q+1}(P L / O) .
$$

Since $\pi_{n}(P L / O) \cong \Theta_{n}$ (see, for example, $\S 6.6$ of [15]), we deduce again that $\tilde{\pi}_{0} \operatorname{Diff}\left(S^{1} \times D^{q}\right.$, rel $\left.\partial\right) \cong \pi_{q}\left(\widetilde{\operatorname{Diff}}\left(S^{1}\right)\right) \cong \Theta_{q+2} \oplus \Theta_{q+1}$.

As I have mentioned above, one could use the same "surgery theory approach" to compute most of the relative mapping class groups of the trivial disk bundles over the spheres. We leave such computations as an exercise for the reader, and return to the "geometric" methods, which also will be used later for computations in the tangent disk bundle case. But first, in the following subsection let me recall some basic definitions and results related to the variation homomorphism, which will play an important role. The reader will find all the corresponding proofs and references in [9]. 
2.3. Kernel of the variation operator. Let $F$ be a parallelizable $2 p$-manifold obtained by gluing $\nu$ handles of index $p \geq 2$ to the $2 p$-disk and rounding the corners:

$$
F=D^{2 p} \cup \bigsqcup_{i=1}^{\nu}\left(D_{i}^{p} \times D^{p}\right)
$$

Evidently, $S^{p} \times D^{p}$ and the closed tangent unit disk bundle $\tau\left(S^{p}\right)$ to $S^{p}$ are examples of such manifolds. Given a diffeomorphism $\varphi$ of $F$ which is the identity on $\partial F$, and a relative cycle $z \in H_{p}(F, \partial F)$, one can consider the variation homomorphism $\operatorname{Var}(\varphi): H_{p}(F, \partial F) \longrightarrow H_{p}(F)$ defined by the formula $\operatorname{Var}(\varphi)[z]:=[\varphi(z)-z]$ (cf. $\S 1,2$ of [23]). The formula $\operatorname{Var}(h \circ g)=\operatorname{Var}(h)+h_{*} \circ \operatorname{Var}(g)$ implies that the isotopy classes of diffeomorphisms that induce trivial variation homomorphisms form a subgroup of $\tilde{\pi}_{0} \operatorname{Diff}(F$, rel $\partial)$. In $\S 2.2$ of [9] we denoted such a subgroup by $\tilde{\pi}_{0} V \operatorname{Diff}(F, \partial)$ and called it the kernel of the variation operator:

$$
\tilde{\pi}_{0} V \operatorname{Diff}(F, \partial):=\left\{\varphi \in \tilde{\pi}_{0} \operatorname{Diff}(F, \partial) \mid \operatorname{Var}(\varphi)[z]=0, \forall[z] \in H_{p}(F, \partial F)\right\} .
$$

Let me also denote by $h$ the homomorphism $\tilde{\pi}_{0} \operatorname{Diff}(\mathcal{D} F) \longrightarrow$ Aut $H_{p}(\mathcal{D} F)$ induced by the natural action of the elements of $\tilde{\pi}_{0} \operatorname{Diff}(\mathcal{D} F)$ on the $p$-th homology of the double. Next claim follows immediately from the proof of Theorem 1 of [9]:

\section{Claim 1.}

Kernel of the homomorphism $h \circ \omega: \tilde{\pi}_{0} \operatorname{Diff}(F$, rel $\partial) \longrightarrow$ Aut $H_{p}(\mathcal{D} F)$ is equal to $\tilde{\pi}_{0} V \operatorname{Diff}(F, \partial)$.

Consider the inclusion $S O(p) \hookrightarrow S O(p+1)$ and denote by $S \pi_{p}(S O(p))$ the image of $\pi_{p}(S O(p))$ under the induced map of homotopy groups $S_{*}: \pi_{p}(S O(p)) \longrightarrow$ $\pi_{p}(S O(p+1))$. Then $S \pi_{6}(S O(6))$ is trivial and for all other $p \geq 3$ the groups $S \pi_{p}(S O(p))$ are given in the table below (see [11], p. 644):

\begin{tabular}{|c|c|c|c|c|c|c|c|c|}
\hline$p(\bmod 8)$ & 0 & 1 & 2 & 3 & 4 & 5 & 6 & 7 \\
\hline$S \pi_{p}(S O(p))$ & $\mathbb{Z}_{2} \oplus \mathbb{Z}_{2}$ & $\mathbb{Z}_{2}$ & $\mathbb{Z}_{2}$ & $\mathbb{Z}$ & $\mathbb{Z}_{2}$ & 0 & $\mathbb{Z}_{2}$ & $\mathbb{Z}$ \\
\hline
\end{tabular}

The following result describes the group $\tilde{\pi}_{0} V \operatorname{Diff}(F, \partial)$ up to extension (see [9], Theorem 3): If $p=2$ then $\tilde{\pi}_{0} V \operatorname{Diff}(F, \partial) \cong\{1\}$, and for all $p \geq 3$ the following sequence is exact

$$
0 \rightarrow \Theta_{2 p+1} \stackrel{\iota_{r}}{\longrightarrow} \tilde{\pi}_{0} V \operatorname{Diff}(F, \partial) \stackrel{\chi_{r}}{\longrightarrow} \operatorname{Hom}\left(H_{p}(F, \partial F), S \pi_{p}(S O(p))\right) \rightarrow 0
$$


The map $\iota_{r}$ is defined in the same way as was done in $\S 2.1$ above. As for the definition of $\chi_{r}$, we refer the reader to $\S 2.2$ of [9].

2.4. The case of $S^{p} \times D^{p}$. Let me now apply the results mentioned above and prove the following

\section{Lemma 3.}

$$
\tilde{\pi}_{0} \operatorname{Diff}\left(S^{p} \times D^{p}, \text { rel } \partial\right) \cong \begin{cases}\mathbb{Z} & \text { if } p=1 \\ \{1\} & \text { if } p=2 \\ \Theta_{2 p+1} \oplus S \pi_{p}(S O(p)) & \text { if } 4 \leq p \text { is even } \\ \Theta_{2 p+1} \oplus S \pi_{p}(S O(p)) \oplus \mathbb{Z} \text { if } 3 \leq p \text { is odd }\end{cases}
$$

Proof. The case of $p=1$ is well known and one can find a proof, for example, in $\S 7$ of [5]. When $p$ is even, the image of

$$
h: \tilde{\pi}_{0} \operatorname{Diff}(\mathcal{D} F) \longrightarrow \text { Aut } H_{p}\left(S^{p} \times S^{p}\right)
$$

is a finite group of order four generated by $\left(\begin{array}{ll}0 & 1 \\ 1 & 0\end{array}\right)$ and $\left(\begin{array}{cc}-1 & 0 \\ 0 & -1\end{array}\right)$ (the first one is induced by the map $(x, y) \longmapsto(y, x))$ and for any $\mu \in \operatorname{Diff}\left(S^{p} \times S^{p}\right)$ with the non-trivial action on the homology there is no non-zero cycle $z \in H_{p}\left(S^{p} \times S^{p}\right)$ such that $\mu_{*}(z)=z$. Since our extended diffeomorphism $\tilde{\varphi}$ preserves $S^{p} \times D_{+}^{p}$ pointwise, it is clear that the group $\tilde{\pi}_{0} \operatorname{Diff}(F$, rel $\partial)$ coincides with the kernel of $h \circ \omega$ and hence, by the claim above, $\tilde{\pi}_{0} \operatorname{Diff}(F$, rel $\partial) \cong \tilde{\pi}_{0} V \operatorname{Diff}\left(S^{p} \times D^{p}, \partial\right)$.

The statement for $p=$ even now follows from the following exact sequence (see [21], Theorem II)

$$
0 \longrightarrow S \pi_{p}(S O(p)) \oplus \Theta_{2 p+1} \longrightarrow \tilde{\pi}_{0} \operatorname{SDiff}\left(S^{p} \times S^{p}\right) \stackrel{B}{\longrightarrow} S \pi_{p}(S O(p)) \longrightarrow 0,
$$

a simple observation (which has been made above) that for every element $[\varphi] \epsilon$ $\tilde{\pi}_{0} V \operatorname{Diff}\left(S^{p} \times D^{p}, \partial\right)$, the element $[\tilde{\varphi}]$ belongs to the kernel of $B$ and exact sequence (1) (the fact that $\tilde{\pi}_{0} V \operatorname{Diff}(F, \partial)=0$, when $p=2$ ).

Assume now that $p$ is odd. Then Aut $H_{p}\left(S^{p} \times S^{p}\right) \cong S L(2, \mathbb{Z})$ when $p$ is 1,3 or 7 , and in the other cases Aut $H_{p}\left(S^{p} \times S^{p}\right)$ is a proper subgroup of $S L(2, \mathbb{Z})$ which consists of the matrices $\left(\begin{array}{l}d_{1} d_{2} \\ d_{3} d_{4}\end{array}\right)$ such that both products $d_{1} d_{2}$ and $d_{3} d_{4}$ 
are even integers ([25], Lemma 5). Clearly, any matrix of this type is congruent modulo 2 either to $I d=\left(\begin{array}{ll}1 & 0 \\ 0 & 1\end{array}\right)$ or $V:=\left(\begin{array}{cc}0 & -1 \\ 1 & 0\end{array}\right)$. We will denote this subgroup by $\Gamma_{V}(2)$. It is well known that $\Gamma_{V}(2)$ is not a normal subgroup of index 3 of $S L(2, \mathbb{Z})$ (see $[20], \S 1.5$ ). Moreover, using the fact that the corresponding

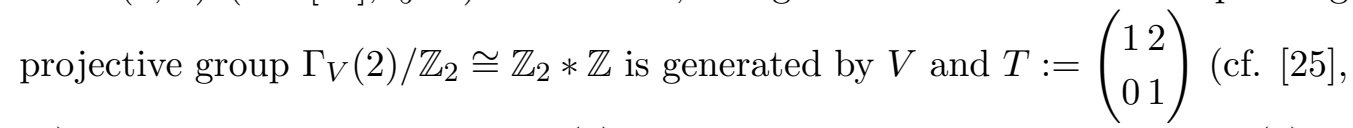
$\S 3)$ one can easily show that $\Gamma_{V}(2)$ admits the following presentation $\Gamma_{V}(2) \cong$ $\left\langle V, T \mid V^{4}=i d, V^{2} T=T V^{2}\right\rangle$. Assume that $p \geq 3$. It follows again from the definition of $\omega$ that the image of $h \circ \omega$ consists of those automorphisms that preserve the class of $H_{p}\left(S^{p} \times S^{p}\right)$ represented by an embedded sphere $S^{p} \times\{*\} \subset$ $S^{p} \times D_{+}^{p} \subset S^{p} \times S^{p}$. If we choose spheres $S^{p} \times\{*\}$ and $\{*\} \times S^{p}$ as the basis $\left\{\left(\begin{array}{l}1 \\ 0\end{array}\right),\left(\begin{array}{l}0 \\ 1\end{array}\right)\right\}$ of $H_{p}\left(S^{p} \times S^{p}\right)$, we see that $\operatorname{Im}(h \circ \omega)$ is generated either by $\left(\begin{array}{ll}1 & 1 \\ 0 & 1\end{array}\right)$

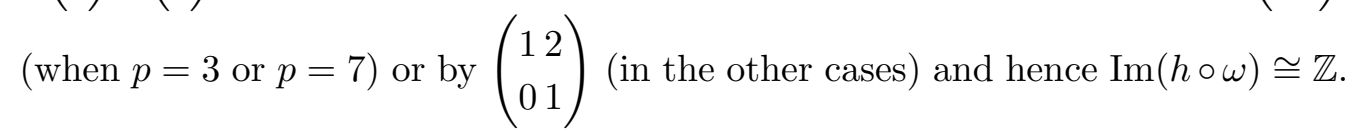
As for the corresponding element of $\tilde{\pi}_{0} \operatorname{Diff}(M$, rel $\partial)$, one again can take the generalized twist $\vartheta$ of $S^{p} \times D^{p}$ defined by the formula $\vartheta(x, y):=(\zeta(y) \circ x, y)$, where $\zeta:\left(D^{p}, S^{p-1}\right) \longrightarrow(S O(p+1), I d)$ is a smooth map which generates image of the map $j_{*}: \pi_{p}(S O(p+1)) \rightarrow \pi_{p}\left(S^{p}\right)$ from the exact homotopy sequence of the fibration $S O(p) \hookrightarrow S O(p+1) \stackrel{j}{\longrightarrow} S^{p}$. To finish the proof we need to show that for $p=$ odd we also have $\tilde{\pi}_{0} V \operatorname{Diff}\left(S^{p} \times D^{p}, \partial\right) \cong \Theta_{2 p+1} \oplus S \pi_{p}(S O(p))$. If $p \geq 5$, one can use exactly the same argument which we gave above for $p=$ even and if $p=3$, it is shown in Example 1 of $[9]$ that $\tilde{\pi}_{0} V \operatorname{Diff}\left(S^{3} \times D^{3}, \partial\right) \cong \Theta_{7} \oplus \mathbb{Z}$.

We now summarize what have been discussed above in the following theorem.

\section{Theorem 1.}

$$
\tilde{\pi}_{0} \operatorname{Diff}\left(S^{p} \times D^{q}, \text { rel } \partial\right) \cong \begin{cases}\mathbb{Z} & \text { if } p=q=1 \\ \{1\} & \text { if } 1 \leq p \leq 2, q=2 \\ \Theta_{p+q+1} \oplus \pi_{p}(S O(p+1)) & \text { if } 3 \leq p=q \\ \Theta_{p+q+1} \oplus \Theta_{q+1} & \text { if } p=1, q \geq 3 \\ \Theta_{p+q+1} \oplus \mathrm{FC}_{q}^{p+1} & \text { if } 1<p<q \\ \Theta_{p+q+1} \oplus \pi_{q}(S O(p+1)) & \text { if } 1 \leq q<p .\end{cases}
$$


Remark: Notice that the homotopy long exact sequence of the fibration $S O(p) \hookrightarrow$ $S O(p+1) \longrightarrow S^{p}$ together with computations of $\pi_{p}(S O(p+1))$ (see $\S 7$ of $[27]$ ) imply that $S \pi_{p}(S O(p)) \cong \pi_{p}(S O(p+1))$, when $p \geq 4$ is even, and $S \pi_{p}(S O(p)) \oplus$ $\mathbb{Z} \cong \pi_{p}(S O(p+1))$, when $p \geq 3$ is odd.

$$
\text { 3. } \tilde{\pi}_{0} \operatorname{Diff}\left(\tau\left(S^{p}\right) \text {, rel } \partial\right)
$$

In this paragraph we discuss the relative mapping class group of the closed tangent unit disk bundle $\tau\left(S^{p}\right)$ to the standard $p$-sphere. Since $\tau\left(S^{p}\right)$ is a stably trivial bundle, its double $\mathcal{D} \tau\left(S^{p}\right)$ will be also diffeomorphic to the product of two spheres $S^{p} \times S^{p}$. However this time $\tau\left(S^{p}\right)$ is not trivial (unless $p=1,3$ or 7 ; [2]) and canonically isomorphic to the normal disk bundle of the diagonally embedded sphere $S^{p} \stackrel{d}{\hookrightarrow} S^{p} \times S^{p}$ ([17], §11). It implies that $d_{*}\left(S^{p}\right)=(1,1) \in \mathbb{Z} \oplus \mathbb{Z} \cong$ $H_{p}\left(S^{p} \times S^{p}\right)$ where, as above, we have chosen $S^{p} \times\{*\}$ and $\{*\} \times S^{p}$ as the basis of $H_{p}\left(S^{p} \times S^{p}, \mathbb{Z}\right)$. Let us assume first that $p$ is odd, the other case will be discussed at the end. Then the self intersection index of the base sphere $S^{p} \hookrightarrow \tau\left(S^{p}\right)$ is zero $\left\langle\left[d\left(S^{p}\right)\right] \cdot\left[d\left(S^{p}\right)\right]\right\rangle=0$ and hence the natural map $j_{*}: H_{p}\left(\tau\left(S^{p}\right) ; \mathbb{Z}\right) \longrightarrow$ $H_{p}\left(\tau\left(S^{p}\right), \partial \tau\left(S^{p}\right) ; \mathbb{Z}\right)$ from the exact sequence of the pair $\left(\tau\left(S^{p}\right), \partial \tau\left(S^{p}\right)\right)$ is zero too. Since any element of $\tilde{\pi}_{0} \operatorname{Diff}\left(\tau\left(S^{p}\right)\right.$, rel $\left.\partial\right)$ has a representative which preserves the boundary pointwise, it follows from this exact sequence that any such element must induce the trivial automorphism of $H_{p}\left(\tau\left(S^{p}\right), \mathbb{Z}\right) \cong \mathbb{Z}$. In particular, any automorphism from $\operatorname{Im}(h \circ \omega)$ must preserve the diagonal class $(1,1)$ and since the determinant of such automorphism is 1 , it is clear that for odd $p$,

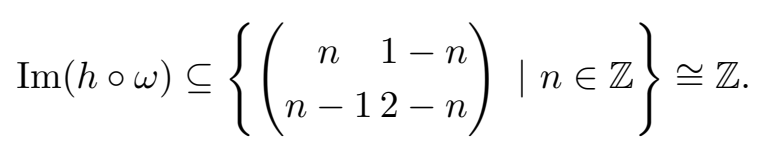

Such matrices form a subgroup of $\Gamma_{V}(2)$ generated by $g:=\left(\begin{array}{cc}2-1 \\ 1 & 0\end{array}\right)=T \cdot V$.

Theorem 2.

$$
\operatorname{Im}(h \circ \omega)=\left\{\left(\begin{array}{rr}
n & 1-n \\
n-12-n
\end{array}\right) \mid n \in \mathbb{Z}\right\}
$$

Proof. Let me denote the tangent unit disk bundle $\tau\left(S^{p}\right)$ by $F$ to be in agreement with $\S 2.3$ above. To prove the theorem, I will present a diffeomorphism 
$\psi \in \operatorname{Diff}(F$, rel $\partial)$, such that $h \circ \omega([\psi])=g^{ \pm 1}$. Consider the hypersurface $H \subset \mathbb{C}^{p+1}$, defined as the zero set of the polynomial $f(z)=z_{0}^{2}+z_{1}^{2}+\ldots+z_{p}^{2}$. This hypersurface has an isolated singular point at the origin. If we denote the intersection of $H$ with a small sphere $S^{2 p+1}$ around the origin by $K$, then the complement $S^{2 p+1} \backslash U(K)$ to the open tubular neighborhood $U(K)$ of $K$ will be a fibration over $S^{1}$ with the fibre $F$ (cf. [14], [16]). Going once around the base circle induces a diffeomorphism (defined up to isotopy and fixing the boundary pointwise) of the fibre which is called the geometric monodromy. I denote this monodromy by $\psi$ and explain why $h \circ \omega([\psi])=g^{ \pm 1}$. Let's pick a point $z \in S^{p}$ on the base sphere of $F$ and take the fiber $D^{p}$ over this point. Clearly, this disk represents a generator of $H_{p}(F, \partial F) \cong \mathbb{Z}$. There is a natural homomorphism $d_{p}: H_{p}(F, \partial F) \longrightarrow H_{p}(\mathcal{D} F)$ which basically assigns to a relative cycle its double in the double $\mathcal{D} F$ (see Definition 2. in [9] for the details), and we denote the image $d_{p}\left(\left[D^{p}, \partial D^{p}\right]\right) \in H_{p}\left(S^{p} \times S^{p}\right)$ by $(a, b)$. If we use $i_{p}$ for the natural homomorphism $H_{p}(F ; \mathbb{Z}) \rightarrow H_{p}(\mathcal{D} F ; \mathbb{Z})$ from the exact sequence of the pair $(\mathcal{D} F, F)$, we deduce from the Picard-Lefschetz formulas (see [1] or [14], (3.1.a)) that $i_{p} \circ \operatorname{Var}(\psi)\left(\left[D^{p}, \partial D^{p}\right]\right)= \pm(1,1)$ depending whether $p \equiv 3(\bmod 4)$ or $p \equiv 1$ (mod 4) respectively. Then using the formula (obtained in [9], Theorem 1.)

$$
\tilde{\psi}_{*} \circ d_{p}=d_{p} \circ I d+i_{p} \circ \operatorname{Var}(\psi) .
$$

we conclude that

$$
\tilde{\psi}_{*}((a, b))= \begin{cases}(a+1, b+1) \text { if } p \equiv 3 & (\bmod 4) \\ (a-1, b-1) \text { if } p \equiv 1 & (\bmod 4)\end{cases}
$$

i.e.

$$
\left(\begin{array}{rr}
n & 1-n \\
n-1 & 2-n
\end{array}\right) \cdot\left(\begin{array}{l}
a \\
b
\end{array}\right)=\left(\begin{array}{l}
a \pm 1 \\
b \pm 1
\end{array}\right) .
$$

These last two equalities imply that $n=0$ or $n=2$ (because the intersection index of $(a, b)$ with the diagonal $(1,1)$ is one) and hence the monodromy corresponds to $g^{ \pm 1}$, and the statement is proven.

Our next corollary follows immediately from this theorem and Claim 1.

\section{Corollary 2.}

When $p>1$ is odd, $\tilde{\pi}_{0} \operatorname{Diff}\left(\tau\left(S^{p}\right)\right.$, rel $\left.\partial\right) \cong \tilde{\pi}_{0} V \operatorname{Diff}\left(\tau\left(S^{p}\right), \partial\right) \oplus \mathbb{Z}$. 
When $p$ is odd and $p \neq 1(\bmod 8)$, we see from the table on page 638 that $S \pi_{p}(S O(p))$ is either trivial or isomorphic to $\mathbb{Z}$. Thus, for such $p$, the exact sequence (1) on page 638 splits and hence the relative mapping class group of $\tau\left(S^{p}\right)$ is $\tilde{\pi}_{0} \operatorname{Diff}\left(\tau\left(S^{p}\right)\right.$, rel $\left.\partial\right) \cong \Theta_{2 p+1} \oplus S \pi_{p}(S O(p)) \oplus \mathbb{Z}$, which is isomorphic to the corresponding group for the trivial $p$-bundle over $S^{p}$. However in general case, sequence (1) has the form

$$
0 \longrightarrow \Theta_{2 p+1} \stackrel{\iota_{r}}{\longrightarrow} \tilde{\pi}_{0} V \operatorname{Diff}\left(\tau\left(S^{p}\right), \partial\right) \longrightarrow S \pi_{p}(S O(p)) \longrightarrow 0
$$

and does not always split. We refer the interested reader to Example 2. from [9], where it is shown that $\exists p=2 m$ such that $S \pi_{p}(S O(p)) \cong \mathbb{Z}_{2}$ and $\tilde{\pi}_{0} V \operatorname{Diff}\left(\tau\left(S^{p}\right), \partial\right)$ $\cong \mathbb{Z}_{4} \oplus \operatorname{Coker}\left(J_{4 m+1}\right)$. In such cases, the group of homotopy spheres that bound parallelizable manifolds is isomorphic to $\mathbb{Z}_{2}$ and $\Theta_{4 m+1} \cong \mathbb{Z}_{2} \oplus \operatorname{Coker}\left(J_{4 m+1}\right)$ (see [4] and [3]).

Moreover, let $\delta$ be a generator of the group $H_{p}\left(\tau\left(S^{p}\right) ; \mathbb{Z}\right)$ when $p=2 m$ and $m \geq 1$. Then it follows again from the Picard-Lefschetz formulas for the geometric monodromy $\psi$ of the quadratic singularity (see [1] or formula (3.3.c) of [14]), that $\psi_{*}(\delta)=-\delta$ (generated by the antipodal map of $S^{p}$ ). Hence we have the following commutative diagram (each row is the same part from the homology sequence of the pair $\left(S^{p} \times S^{p}, \tau\left(S^{p}\right)\right)$ and vertical maps are induced by the geometric monodromy $\psi$ ):



Since $d_{*}(1)=(1,1), \tilde{\psi}_{*} \in \mathbb{Z}_{2} \times \mathbb{Z}_{2}$, and the map $\tilde{\psi}$ preserves the anti-diagonal $\left\{(x,-x) \in S^{p} \times S^{p}\right\}$ pointwise, we obtain

$$
\tilde{\psi}_{*}=\left(\begin{array}{cc}
0 & -1 \\
-1 & 0
\end{array}\right) .
$$

This gives the following exact sequence when $p$ is even:

$$
0 \longrightarrow \tilde{\pi}_{0} V \operatorname{Diff}\left(\tau\left(S^{p}\right), \partial\right) \longrightarrow \tilde{\pi}_{0} \operatorname{Diff}\left(\tau\left(S^{p}\right), \text { rel } \partial\right) \longrightarrow \mathbb{Z}_{2} \longrightarrow 0 .
$$


It is mentioned in [9] (see Example 2.) that this sequence (2) does not split in general as well, and when the Kervaire sphere $\Sigma \in \Theta_{2 p+1}$ is exotic and $m \neq 0$ $(\bmod 4)$, we have $\tilde{\pi}_{0} \operatorname{Diff}\left(\tau\left(S^{p}\right)\right.$, rel $\left.\partial\right) \cong \mathbb{Z}_{8} \oplus \operatorname{Coker}\left(J_{4 m+1}\right)$.

\section{REFERENCES}

[1] V. Arnold, S. Gusein-Zade, A. Varchenko: Singularities of Differentiable Maps, Vol II, Monographs in Mathematics 83, Birkhäuser, 1988

[2] R. Bott, J. Milnor: On the parallelizability of the spheres, Bulletin of the A.M.S. 64 (1958), $87-89$.

[3] W. Browder: The Kervaire invariant of a framed manifold and its generalization, Annals of Math. 90 (1969), 157-186.

[4] G. Brumfiel: On the homotopy groups of BPL and PL/O, III, Michigan Math. Journal 17 (1970), 217-224.

[5] H. Gluck: The embedding of two-spheres in the four-sphere, Transactions of the AMS 104 (1962), 308-333.

[6] C. McA. Gordon: Knots, homology spheres, and contractible 4-manifolds, Topology 14 (1975), 151-172.

[7] A. Haefliger: Plongements différentiables dans le domaine stable, Comment. Math. Helv. 37 1962/1963 155-176.

[8] A. Haefliger: Differentiable embeddings of $S^{n}$ in $S^{n+q}$ for $q>2$, Annals of Math. 83 (1966), 402-436.

[9] L. Kauffman, N. Krylov: Kernel of the variation operator and periodicity of open books, Topology Appl. 148 (2005), no. 1-3, 183-200.

[10] M. Kervaire, J. Milnor: Groups of homotopy spheres: I, Annals of Math. 77 (1963), 504-537.

[11] M. Kreck: Isotopy classes of diffeomorphisms of $(k-1)$-connected almost parallelizable $2 k$-manifolds, Algebraic topology, Aarhus 1978, 643-663, Lecture Notes in Math., vol.763, Springer, Berlin, 1979.

[12] J. Levine: A classification of differentiable knots, Annals of Math. 82 (1965), 15-50.

[13] J. Levine: Self-equivalences of $S^{n} \times S^{k}$, Transactions of the AMS 143 (1969), 523-543.

[14] E. Looijenga: Isolated singular points on complete intersections, London Mathematical Society Lecture Note Series, 77. Cambridge University Press, Cambridge, 1984.

[15] W. Lück: A basic introduction to surgery theory, Topology of high-dimensional manifolds, No. 1, 2 (Trieste, 2001), 1-224, ICTP Lect. Notes, 9, Abdus Salam Int. Cent. Theoret. Phys., Trieste, 2002.

[16] J. Milnor: Singular Points of Complex Hypersurfaces, Ann. of Math. Studies, No. 61, Princeton University Press, 1968.

[17] J. Milnor, J. Stasheff: Characteristic classes, Ann. of Math. Studies, No. 76, Princeton University Press, 1974. 
[18] J. Munkres: Elementary differential topology, Ann. of Math. Studies, No. 54, Princeton University Press, 1966.

[19] F. Quinn: A geometric formulation of surgery, 1970 Topology of Manifolds (Proc. Inst., Univ. of Georgia, Athens, Ga., 1969) pp. 500-511 Markham, Chicago, Ill.

[20] R. Rankin: Modular forms and functions, Cambridge University Press, 1977.

[21] H. Sato: Diffeomorphism groups of $S^{p} \times S^{q}$ and exotic spheres, Quart. J. Math. Oxford (ser. 2) 20 (1969), 255-276.

[22] H. Sato: Diffeomorphism groups and classification of manifolds, J. Math. Soc. Japan 21 (1969), 1-36.

[23] J. Stevens: Periodicity of branched cyclic covers of manifolds with open book decomposition, Math. Ann. 273 (1986), 227-239.

[24] E. Turner: Diffeomorphisms of a product of spheres, Invent. Math. 8 (1969), 69-82.

[25] C.T.C. Wall: Killing the middle homotopy groups of odd dimensional manifolds, Transactions of the AMS 103 (1962), 421-433.

[26] C.T.C. Wall: Classification problems in differential topology. I-II, Topology 2 (1963), 253272.

[27] C.T.C. Wall: Classification problems in differential topology. III. Applications to special cases, Topology 3 (1965), 291-304.

[28] C.T.C. Wall: Surgery on compact manifolds, Second edition. Edited by A. A. Ranicki. Mathematical Surveys and Monographs, 69. AMS, Providence, RI, 1999.

[29] M. Weiss, B. Williams: Automorphisms of manifolds, Surveys on surgery theory, Vol. 2, 165-220, Ann. of Math. Stud., 149, Princeton Univ. Press, Princeton, NJ, 2001.

Nikolai A. Krylov

International University Bremen

School of Engineering \& Science

P.O. Box 750 561, 28725 Bremen, Germany

E-mail: n.krylov@iu-bremen.de

Current address:

Siena College, School of Science

515 Loudon road

Loudonville, NY 12211, USA

E-mail: nkrylov@siena.edu 